\title{
Karpale Instabilität
}

\author{
Christian Voigt
}

\section{Zusammenfassung}

Die Instabilität der Handwurzel ist eine schwere, immer behandlungsbedürftige Verletzung. Die beiden Reihen der Handwurzelknochen werden zusammen mit ihren Bandverbindungen als Ringstruktur gesehen. Kommt es zu einer Unterbrechung dieses Ringes, resultiert eine Instabilität in der Handwurzel. Die Instabilität führt dann zu typischen Veränderungen der Positionen der Handwurzelknochen zueinander und zum Zusammenbruch der Höhe der Handwurzel. Ist dieser Zustand einmal eingetreten, so ist eine spezifische Behandlung nicht mehr möglich. Dadurch resultiert letztlich eine Zerstörung des Handgelenkes. Als einzigen Rückzug gibt es dann die teilweise oder vollständige Versteifung des Handgelenkes. Um diese schweren Verletzungsfolgen zu vermeiden, muss nach einer Verletzung im Handgelenksbereich die Instabilität ausgeschlossen werden. Dazu sind geeignete Untersuchungstechniken und besonders auch exakte Röntgenaufnahmen in Standardprojektionen erforderlich. Eventuell hilft die Durchleuchtung. In diesem Zusammenhang ist besonders darauf hinzuweisen, dass auch Verletzungen wie der körperferne Speichenbruch von einer karpalen Instabilität begleitet sein können, aber auch die ossäre Unterbrechung des Ringes der Handwurzelknochen bei Fraktur von zusätzlichen ligamentären Verletzungen.

\section{Einleitung}

Die häufigste Fraktur des Menschen ist der Bruch des körperfernen Endes der Speiche. Der junge Assistent sieht im Rahmen seiner Tätigkeit bei der Erstversorgung von Patienten mehrfach täglich Röntgenbilder des Handgelenkes. Dabei wird trainiert, die distalen Radiusfrakturen zu erkennen und nach der Diagnostik die richtige Therapie einzuleiten.

Im Medizinstudium wird die Anatomie am Anfang gelehrt und meist zentrifugal gelernt. Die anatomischen Kenntnisse von Schulter und Oberarm sind meist sehr gut, am Unterarm verdämmert das Wissen, am Handgelenk ist im knöchernen Niveau nicht selten die Kenntnis entsprechend der Abb.1.

OP-JOURNAL 2001; 17: 258-262

(c) Georg Thieme Verlag Stuttgart · New York
Auch wenn durch Merksätze die Beziehung der Handwurzelknochen zueinander gelernt werden soll, ist häufig die Umsetzung im Röntgenbild nur unzureichend.

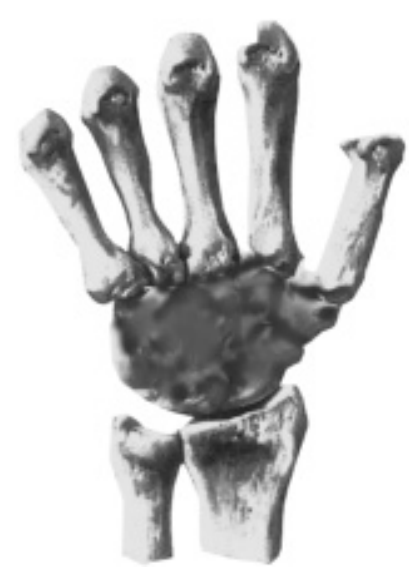

Abb.1 Nicht selten vorhandene Vorstellung von der Anatomie der Handwurzel.
Ausgerechnet in diesem Bereich des mangelhaften Wissens ereignen sich schwere Verletzungen, die zu starken Funktionsbehinderungen der betroffenen Hand führen können.

\section{Definitionen und Anatomie}

Die körpernahe und körperferne Reihe der Handwurzelknochen wird zusammen mit den sie verbindenden Bändern funktionell als Ringstruktur gesehen. Dieser Ring ist bei der Kraftüberleitung von der Hand zum Unterarm großen Belastungen ausgesetzt. Gleichzeitig wird über ihn die Beweglichkeit des Handgelenkes vermittelt. Seine Stellung ist also nicht nur anatomisch gesehen zentral. Wird dieser Ring nun unterbrochen, resultiert eine Instabilität.

Die Unterbrechung kann ligamentär, ossär oder kombiniert sein.

Eine ligamentäre Instabilität tritt dann auf, wenn zwischen Handwurzelknochen befindliche Bandstrukturen zerrissen sind und eine unnatürliche Beweglichkeit zwischen diesen Handwurzelknochen auftritt. Häufigstes Beispiel hierfür ist die Verletzung des Bandapparates zwischen dem Kahnbein und dem Mondbein.

Die ossäre Instabilität ist Folge eines Bruchs einer der Handwurzelknochen. Die häufigste Verletzung betrifft hier das Kahnbein.

Bei den kombiniert ligamentären und ossären Verletzungen handelt es sich um Verrenkungsbrüche im Handwurzelbereich, häufig läuft die Bruchlinie durch das Kahnbein, die Luxation findet um das Mondbein herum statt. Diese Verrenkungsbrüche sind häufig Folge eines Rasanztraumas. Der nicht selten schwerverletzte Patient erhält ungeteilte fachkompetente Aufmerksamkeit und somit wird in aller Regel diese Verletzung kompetent und rasch diagnostiziert und der Behandlung zugeführt. 
Bei den rein ligamentären bzw. ossären Instabilitäten kommt es nicht selten zu einem Übersehen dieser Verletzungen, nicht zuletzt aus den oben angeführten Gründen des mangelhaften Wissens.

Im weiteren Verlauf resultiert dann eine Veränderung der Stellung der Handwurzelknochen zueinander. Aufgrund der übergeleiteten Kräfte, der Ebenen der Artikulationsflächen und der Krafteinwirkung bei Bewegung und Belastung, treten typische Fehlstellungen besonders um das Kahnbein und um das Mondbein bzw. Kopfbein auf. Letztendlich wird der gesamte Handwurzelbereich zerrüttet, es tritt der karpale Kollaps ein, die Länge der Handwurzel wird verringert, Arthrosen bilden sich aus, die Beweglichkeit sinkt und die Kraftausübung der betroffenen Hand wird stark limitiert.

Wenn dieser Zustand eingetreten ist, ist eine spezifische Therapie der ursprünglich übersehenen Handwurzelverletzungen nicht mehr möglich. Rückzug bietet nur noch eine Teilversteifung im Handwurzelbereich oder gar die vollständige Versteifung zwischen Radius und Handwurzel mit den gut vorstellbaren Einschränkungen im täglichen Leben und im Beruf.

\section{Verletzung 1}

\section{Skapholunäre (SL) Dissoziation}

Die Diagnostik der Bandverletzungen zwischen dem Os scaphoideum und Os lunatum setzt exakte Röntgenaufnahmen des Handgelenkes in 2 Ebenen in Standardprojektionen voraus.

Ist die Distanz zwischen beiden Knochen größer als $2 \mathrm{~mm}$ in der Aufsicht, kann von einer vollständigen Zerreißung der Bänder ausgegangen werden. Die Verletzung betrifft dann aber auch andere karpale Strukturen, denn es konnte bei arthroskopischen Untersuchungen gezeigt werden, dass eine bloße Durchtrennung der Bänder zwischen Kahnbein und Mondbein noch keine Instabilität bewirkt.

In der Seitansicht findet sich ein typisches Verkippen des Os lunatum, indem seine zum Os capitatum gewandte Gelenkfläche nach handrückenwärts zu blicken beginnt. Ursache hierfür ist neben der Bandverletzung der etwas exzentrische Druck des Os capitatum auf die mehr dorsal liegenden Gelenkflächenanteile am Os lunatum.
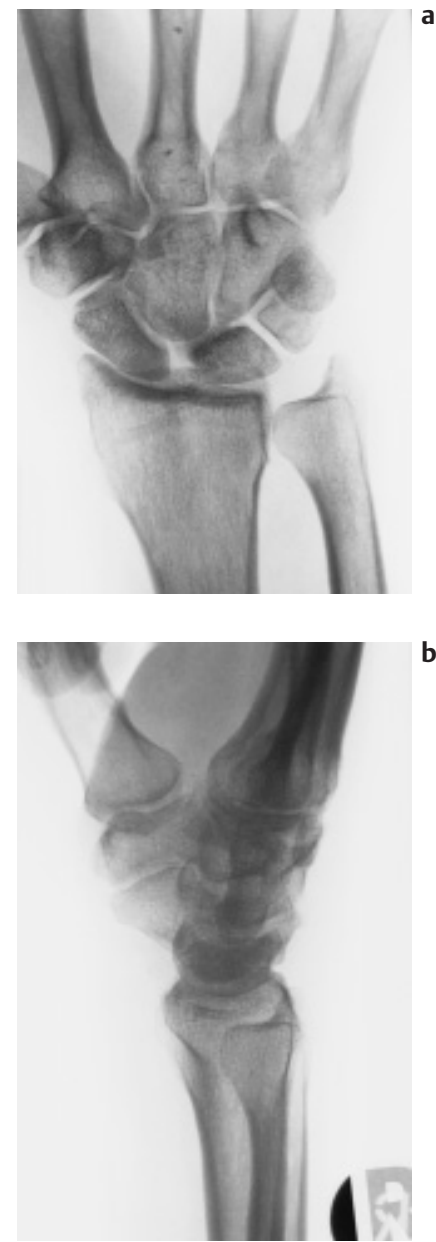

b

Abb.2au.b Skapholunäre Dissoziation, in der Aufsicht gut an der Distanz zwischen Os lunatum und Os scaphoideum sichtbar, in der seitlichen Projektion unauffällige Verhältnisse.

Es gibt aber auch Situationen, in denen in der Aufsicht eine regelrechte Stellung zwischen Kahnbein und Mondbein besteht, beim Bewegen jedoch das Kahnbein seine Position verlässt und aufgrund der fehlenden Bandverbindungen verkippt. Diese dynamische SL-Dissoziation ist nur dann zu diagnostizieren, wenn gezielt nach ihr gesucht wird.

Die Behandlung der SL-Dissoziation kann offen oder minimalinvasiv perkutan erfolgen.

Bei der offenen Behandlung werden durch eine dorsale Inzision Kahnbein und Mondbein dargestellt. Man kann in beide Knochen einen Bohrdraht einbringen, über den diese dann manipulierbar sind. Die eigentliche Bandverletzung ist selten nähbar, häufig ist eine transossäre Fixierung des abgerissenen Bandstumpfes erforderlich. Bei veralteten Bandver-
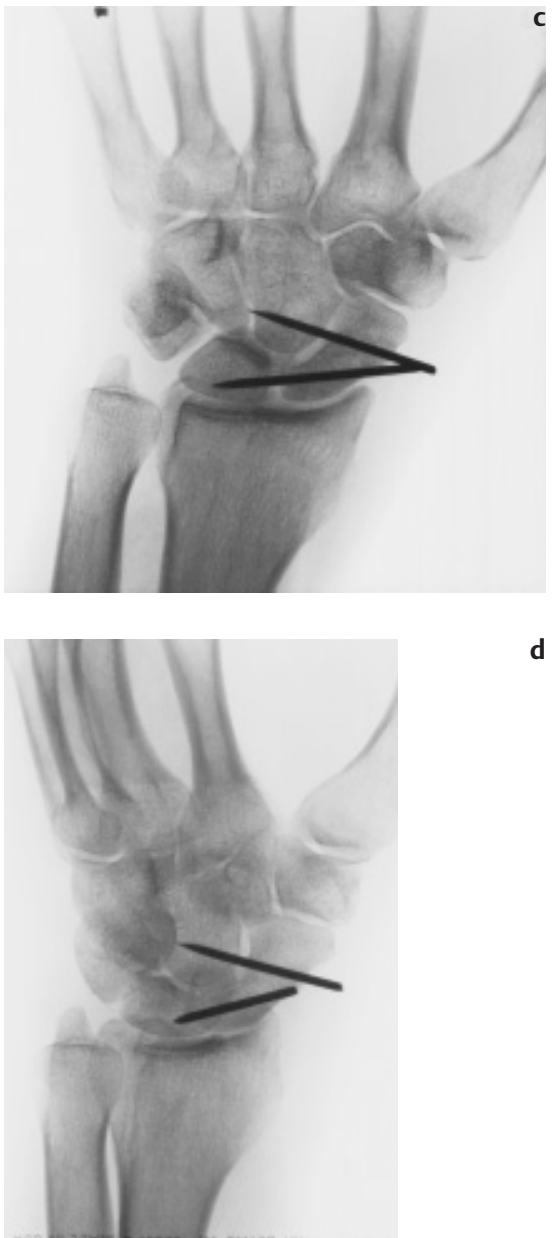

Abb.2cu.d Nach offener Reposition und Adaptierung der Bänder Transfixation des Os scaphoideum zum Os lunatum und Os capitatum. In der Aufsicht nun regelrechter Abstand zwischen Kahnbein und Mondbein.

letzungen kann auch eine Bandplastik mit Sehne des Musculus palmaris longus oder Teilsehne, beispielsweise des Musculus extensor carpi radialis brevis, erfolgen.

Obligatorisch ist die Transfixierung des Gelenkes zwischen Kahnbein und Mondbein durch Bohrdraht, um die Stellung der Handwurzelknochen gegeneinander halten zu können (Abb.2).

Die postoperative Weiterbehandlung erfolgt durch Unterarmgips für 6 Wochen, dann kann auch eine Bohrdrahtentfernung durchgeführt werden (Hahn 1999).

Bei der minimalinvasiven Technik wird perkutan vorgegangen und das Ergebnis arthroskopisch überwacht. Bei frischen Verletzungen wird davon ausgegangen, dass die Bandenden suffizient verheilen, wenn sie einander genähert sind und in 

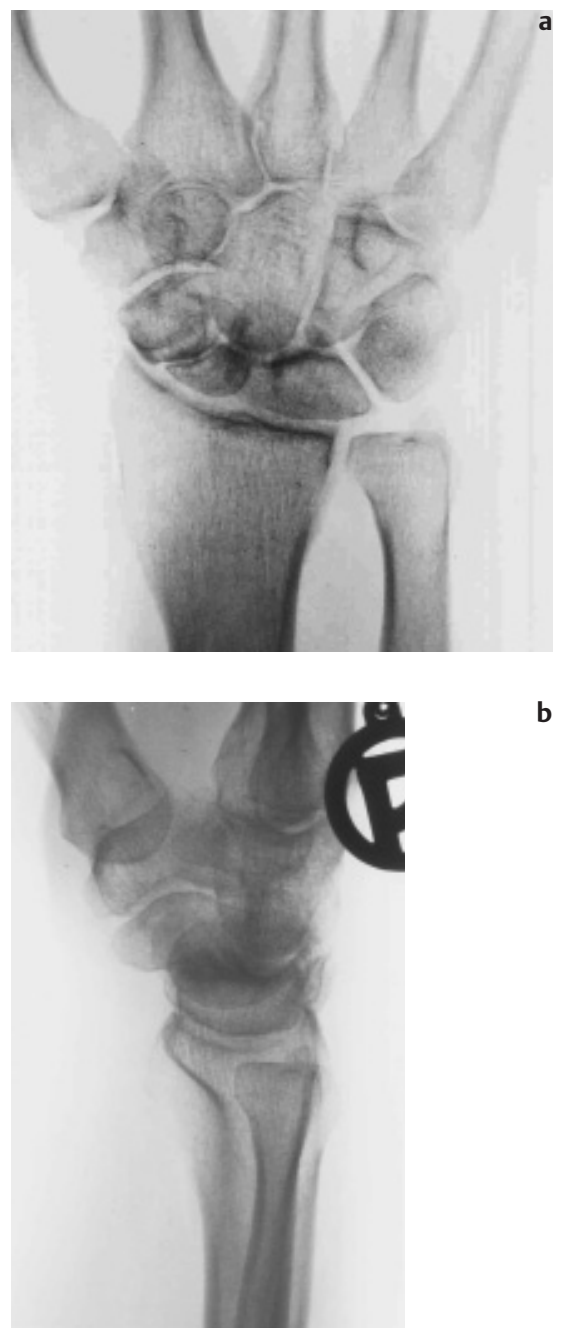

Abb.3au.b SNAC-Handgelenk. Pseudarthrose des Kahnbeins mit Verkippung des distalen Anteils in der Aufsicht gut sichtbar. Seitlich typisches Kippen des Mondbeins nach handrückenseits.

Kontakt kommen. Auch hier wird temporär das Gelenk transfixiert, die regelrechte Stellung der Handwurzelknochen zueinander ist im Röntgenbild während der Operation sichtbar und kann durch die Arthroskopie zusätzlich überprüft werden (Schädel-Höpfner 2000).

Sollte eine zusätzliche Instabilität zum Os capitatum vorliegen, so wäre auch hier eine Transfixierung erforderlich. Die postoperative Weiterbehandlung besteht ebenfalls durch 6-wöchige Gipsanlage.

Wird die SL-Dissoziation übersehen, resultiert eine schwerwiegende Fehlstellung im gesamten Karpus mit Zusammenbruch der Stellung der Handwurzelknochen gegeneinander. Diese Veränderung wird - abgeleitet aus der Verletzung und den Folgen - als SLAC-Handge-

$b$

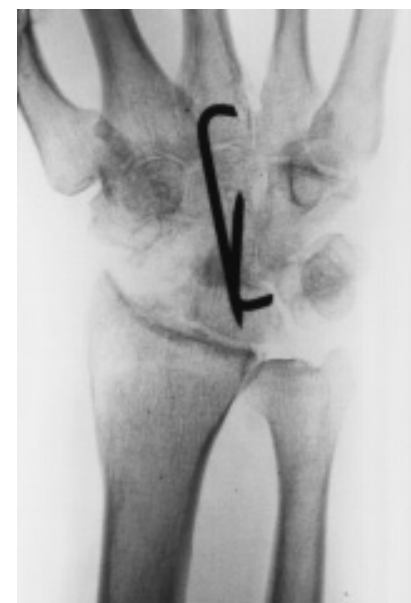

C

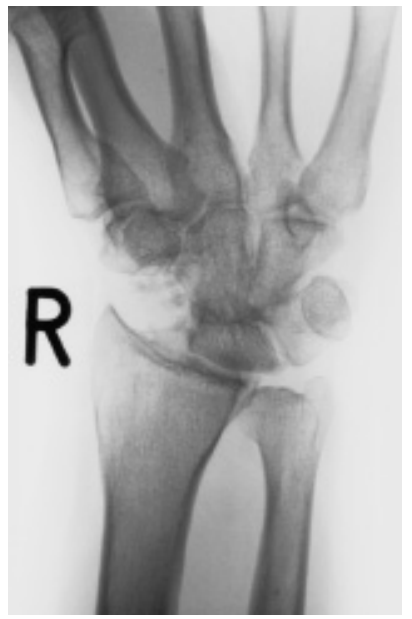

e
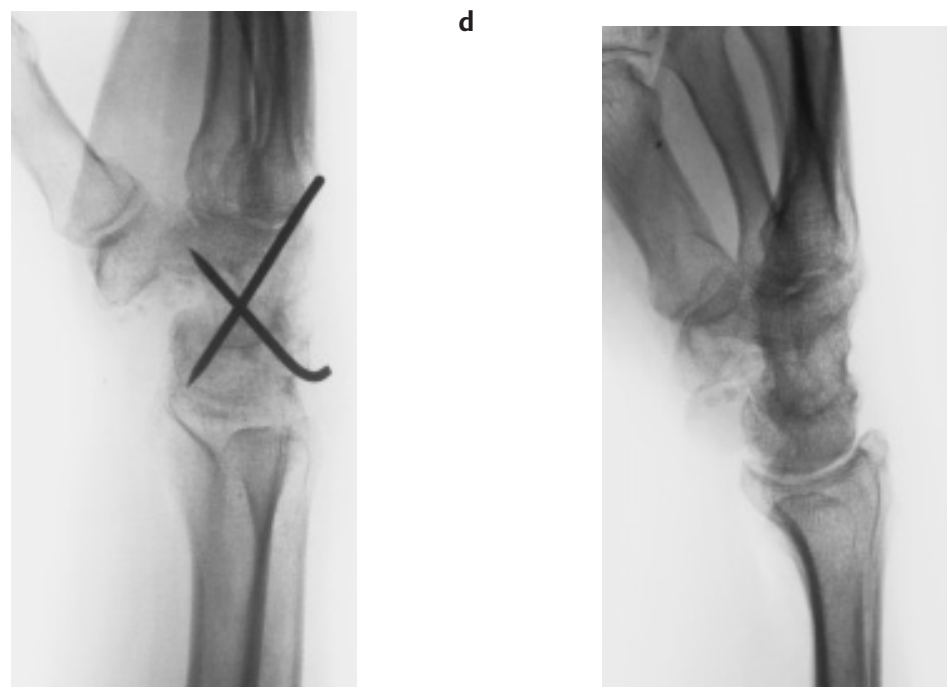

f

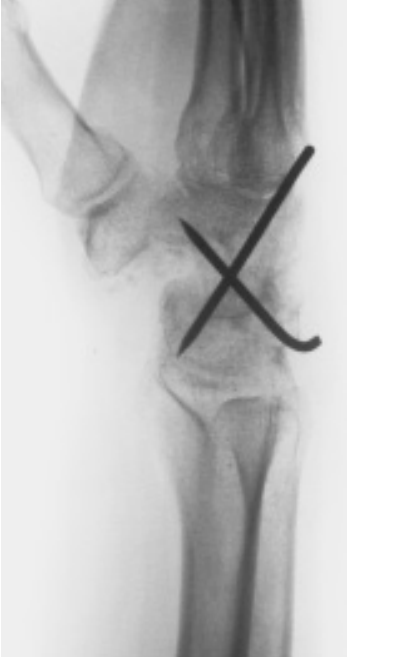

Abb.3cu.d Nach Entfernung des Kahnbeins mediokarpale Arthrodese mit Bohrdrahtfixierung, zusätzliche Gipsruhigstellung erforderlich.

lenk bezeichnet. Dahinter verbirgt sich der Ausdruck „Scapholunate advanced collaps" (Krimmer et al.).

Ist dieser Zustand einmal eingetreten, so lassen auch bandplastische Maßnahmen keine Rettung des Handgelenkes mehr erwarten. Je nach Ausprägung und Ort der aufgetretenen interkarpalen bzw. radiokarpalen Arthrose, wird eine mediokarpale Teilversteifung oder eine Gesamtversteifung radio-interkarpal erforderlich werden.

\section{Verletzung 2}

\section{Skaphoidfraktur}

Beim Bruch des Kahnbeins tritt die knöcherne Instabilität des Karpus auf. Das Kahnbein ist bei Bewegung des Handge-
Abb.3 eu.f Ausheilung. In beiden Ebenen ist die Höhe des Karpus rekonstruiert, die Kippung des Mondbeins ist beseitigt.

lenkes erheblichen Kräften ausgesetzt, die bei nicht intaktem Knochen zu einer Verkippung des distalen zum proximalen Teil des Skaphoids führt. Dadurch kommt es im Verlauf zu einer typischen Gefügestörung in der Handwurzel, die schließlich in das SNAC Handgelenk (Scaphoid nonunion advanced collaps) mündet.

Wie bei der SL-Dissoziation ist eine spezifische Therapie beim Vollzustand dieser Verletzungsfolge nicht mehr möglich. Als Ausweg gilt auch hier die radio-karpale Arthrodese oder die inter-karpale Teilarthrodese (Abb.3).

Die spezifische Therapie der Skaphoidfraktur wird in einem anderen Beitrag dieses Heftes des OP-Journales abgehandelt. Deshalb erfolgt hier keine besondere Darstellung der operativen Therapie. 


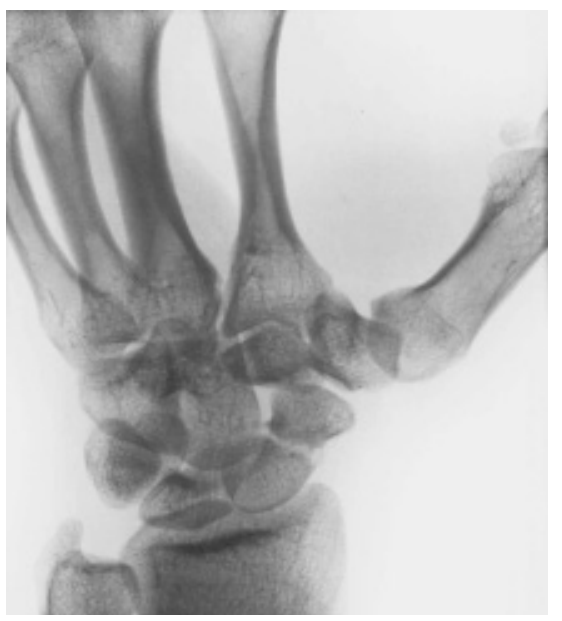

Abb. 4a Kahnbeinbruch.

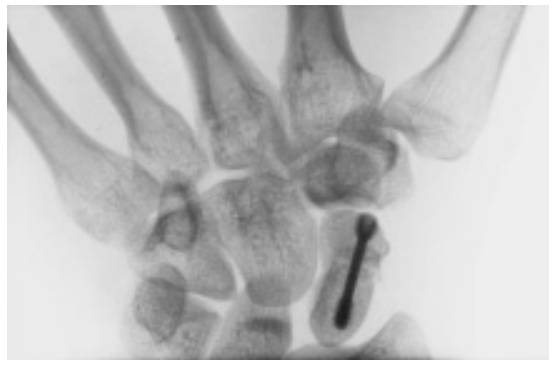

Abb.4b Nach Versorgung mit HerbertSchraube deutliches Klaffen zwischen Kahnbein und Mondbein.

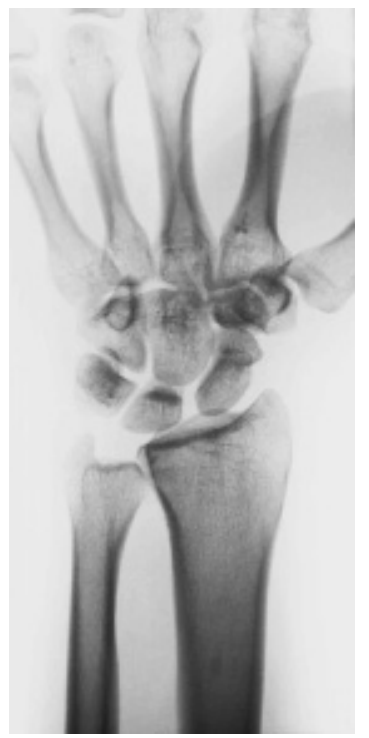

Abb.4c Schon in der ap-Projektion der Unfallaufnahme ist die vergrößerte Distanz zwischen Kahn- und Mondbein deutlich sichtbar.

Nicht unerwähnt sollte jedoch die Tatsache bleiben, dass trotz Auftretens einer Fraktur des Kahnbeines zusätzliche ligamentäre Verletzungen wie die SL-Dissoziation bestehen können.

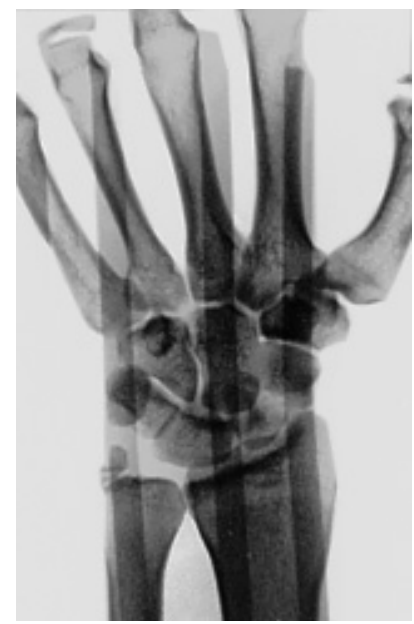

a

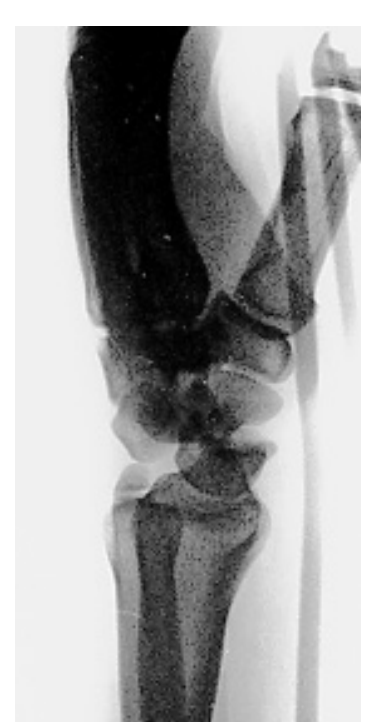

Abb.5au.b Perilunäre Handwurzelluxation. In der Aufsicht schlechte Abgrenzbarkeit zwischen Os lunatum und Os capitatum. Seitlich sieht man das in seiner Position verbliebene Mondbein, das wegen der Luxation der übrigen Karpalknochen nach dorsal in die Handinnenfläche blickt.

Die Abb. 4 zeigt die schon auf der ursprünglichen a.p. Aufnahme sichtbare SL-Dissoziation. Die halbschräge Projektion zeigt die Skaphoidfraktur auch nach Versorgung der Skaphoidfraktur, ist weiterhin eine SL-Dissoziation vorhanden, die dann sekundär versorgt wurde.

\section{Verletzung 3}

\section{Perilunäre Luxation}

Die Diagnostik der perilunären Handwurzelluxation ist durch Standard-Röntgenaufnahmen des Handgelenkes in zwei senkrecht aufeinander stehenden Ebenen leicht möglich. Dennoch ist festzuhalten, dass nach Angaben mancher Autoren
(Saffar 1988) bis zur Hälfte dieser Verletzungen zu spät diagnostiziert werden.

Ursache für die späte Diagnose ist dann meist das sich entwickelnde Karpaltunnelsyndrom durch Einengung des Karpalkanales wegen der häufig nach dorsal auftretenden Luxation der übrigen Karpalknochen um das Os lunatum herum.

Was ist die Ursache für diese diagnostischen Mängel?

Leider sieht man häufig retrospektiv auf den regelrechten Röntgenaufnahmen die gesamte Verletzung. Es liegt also eine mangelhafte Kenntnis der Röntgen-Anatomie vor. Jeder in der Erstversorgung von Patienten tätige Arzt sollte ausreichend trainiert sein, um diese Verletzungen nicht zu übersehen.

Manchmal sind aber auch die Projektionen nicht standardmäßig angefertigt, d.h. es liegt weder eine exakte Aufsicht noch eine exakte Seitansicht im Handwurzelbereich vor.

Bei halbschrägen Projektionen kann dann der Ungeübte - manchmal aber auch der Erfahrene - diese Verletzungen übersehen, da die regelrechte Artikulation zwischen Os lunatum und Os capitatum nicht nachvollziehbar ist. Deshalb sollten alle Röntgenaufnahmen, die nicht eindeutige a.p.- und seitliche Projektionen wiedergeben, solange wiederholt werden, bis eine regelrechte Auswertung möglich ist (Abb. 5).

Bei der Therapie der perilunären Luxation ist ein einmaliger Repositionsversuch in Narkose erlaubt. Brüskere Repositionsmanöver sind zu vermeiden, da es sonst zu Läsionen des Nervus medianus und auch zu Knorpelläsionen am Mondbein kommen kann.

Nach der geschlossenen Reposition ist unbedingt die Stabilität skapholunär zu überprüfen. Liegt eine SL-Dissoziation vor, muss diese unmittelbar therapiert werden. Bei bloßer Luxation ohne SL-Dissoziation wird dann im Unterarm-Skaphoidgips für 6 Wochen weiterbehandelt.

Ist eine geschlossene Reposition nicht möglich, so wird über einen palmaren $\mathrm{Zu}$ gang die Situation dargestellt, im eigenen Vorgehen wird gleichzeitig der Karpalkanal gespalten. Nach Reposition der Handwurzel zum Os lunatum hin können in der Tiefe des Karpalkanales liegende Bandstrukturen durch Nähte adaptiert werden. 


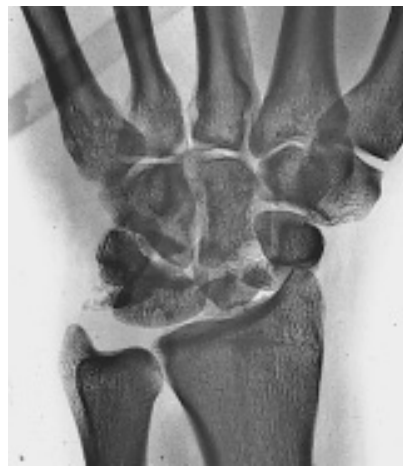

Abb.6a Aufsicht bei transskaphoido-perilunärer Luxationsfraktur. Deutlich sichtbare Trümmerung des Kahnbeins, Verkürzung der Höhe des Karpus und Projektion mehrerer Knochenanteile im Bereich des Mondbeines übereinander.

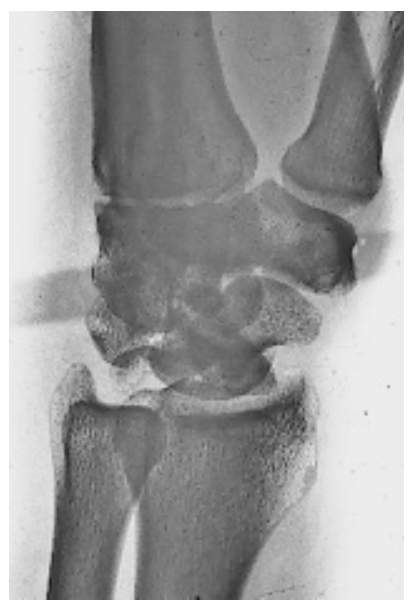

Abb.6b Misslungene seitliche Projektion (tatsächlich halbschräg), Verletzungsausmaß deshalb nicht genau feststellbar, nur die verringerte Höhe des Karpus fällt auch hier auf.

Auch hier muss die SL-Dissoziation ausgeschlossen werden, liegt sie vor, ist ihre Therapie zwingend erforderlich. Nach dem offenen Vorgehen ist ebenfalls eine Gipsbehandlung von 6 Wochen im Unterarm-Skaphoidgips erforderlich.

\section{Verletzung 4}

\section{Transskaphoido-perilunäre Luxationsfraktur}

Ursache für diese schwere Handgelenksverletzung ist in aller Regel ein erhebliches Dezelerationstrauma. Aufgrund der Größe der Krafteinwirkung sind häufig andere Regionen des Körpers mitverletzt, wenn der Patient nicht gar polytraumatisiert ist. Aus diesem Grunde wird ihm die ungeteilte Aufmerksamkeit von viel Fachkompetenz zuteil. Deshalb werden - vorausgesetzt, entsprechende Röntgenaufnahmen wurden angefertigt - diese Verletzungen seltener übersehen.

Auch hier ist zunächst eine Röntgenaufnahme des Handgelenkes in der Aufsicht und einer exakt seitlichen Projektion zu fordern. Mit diesen beiden Abbildungen ist die Diagnose eindeutig zu stellen.

Im Beispiel ist die Aufsicht gut projiziert, die Seitansicht ist verdreht, nur der Erfahrene vermag das Ausmaß der Verletzung sofort zu erkennen (Abb.6). Leider sind solche Röntgenaufnahmen gerade in der Hektik der Versorgung Schwerverletzter nicht selten.

Die Therapie der hochgradig instabilen Handwurzelverletzungen besteht in der schrittweisen Wiederherstellung der zerstörten Strukturen. Dazu ist immer ein offenes Vorgehen erforderlich. Je nach Verletzung ist ein palmarer oder ein dorsaler Zugang zum Handgelenk zu wählen. Nach Beseitigung der Luxation muss die Fraktur des Skaphoids in suffizienter Weise behandelt werden. Es empfiehlt sich das Einbringen einer HerbertSchraube. Durch diese Maßnahme ist die zuvor noch vorhandene Instabilität plötzlich beseitigt. Spontane Luxationen, die sonst während der Operation sichtbar sind, treten nicht mehr auf. Danach sind Bandverletzungen $\mathrm{zu}$ diagnostizieren und eine entsprechende Transfixation der Gelenkpartner vorzunehmen. Adaptierende Bandnähte ergänzen das Vorgehen.

Trotz der scheinbaren Stabilität nach Versorgung des Skaphoids muss mit Gips weiterbehandelt werden, um die schweren ligamentären Verletzungen ausheilen zu lassen. Liegen zusätzliche Frakturen beispielsweise des Prozessus styloideus radii oder am Ellengriffel vor, so muss auch hier operativ interveniert werden, um insgesamt eine Stabilität des Karpus wieder zu erreichen.

\section{Literatur}

1 Hahn P, Krimmer H, Prommersberger K-J, van Schoonhoven J. Die skapholunäre Dissoziation. Ergebnisse nach Bandnaht und Bandplastik. Handchir Mikrochir Plast Chir 1999; 31: 346-350

2 Schädel-Höpfner M, Böhringer G, Gotzen L. Ergebnisse nach minimal-invasiver Therapie der akuten skapholunären Dissoziation. Handchir Mikrochir Plast Chir 2000; 32 : $333-338$

3 Saffar P. Perilunar dislocations of the wrist In Razemon JP und Fisk GR: The wrist. Churchill Livingstone Edinburgh 1988

${ }^{4}$ Krimmer H, Krapohl B, Sauerbier M, Hahn P. Der posttraumatische karpale Kollaps (SLAC- und SNAC-wrist) - Stadieneinteilung und therapeutische Möglichkeiten. Handchir Mikrochir Plast Chir 1997; 29: 228 - 233

Prof. Dr. med. Christian Voigt Chefarzt der Klinik für Unfall-, Handund Wiederherstellungschirurgie

Städtisches Klinikum Solingen

Gotenstr. 1

D-42653 Solingen 